\title{
Effective Mass Layer of a Single Drop of Liquid Located on a Quartz Crystal Microbalance
}

\author{
Qingsong Bai and Xianhe Huang* \\ University of Electronic Science and Technology of China, Chengdu 611731, China
}

(Received July 11, 2016; accepted December 6, 2016)

Keywords: quartz crystal microbalance, effective mass layer, mass sensitivity, decay length

A model describing the frequency response of a quartz crystal microbalance (QCM) loaded with a single drop of liquid was established. The frequency shift of a QCM loaded with a single drop of liquid depends on only the effective mass layer instead of the total mass of the liquid droplet, and the effective mass layer is relevant to the droplet radius, the operating frequency of the QCM, and the density and viscosity of the liquid. In addition, a novel verification experiment was designed and performed, which indicated the validity of this model.

\section{Introduction}

Quartz crystal microbalances (QCMs) have recently attracted much attention as promising label-free, inexpensive, and highly sensitive microsensors capable of performing in liquid environments. ${ }^{(1-3)}$ Commonly used in practical applications are the AT-cut quartz crystal resonator in which thickness-shear vibrations are excited to set up standing waves. The underlying principle is to translate mass adsorption on a QCM surface into resonance frequency shift, which can be precisely and readily detected. Recent research studies using QCMs have demonstrated wide applications of this ultrasensitive sensor in various areas of science and technology. ${ }^{(4-11)}$ The linear relationship between the mass change and the resonance frequency shift of a QCM is described using the Sauerbrey equation. ${ }^{(12)}$ Kanazawa developed an improved model to describe the response of a QCM performing in a Newton liquid. ${ }^{(13)}$

QCMs are typically investigated for sensor applications in which one surface of a QCM is completely immersed in a Newtonian liquid, or usually used as a core detector to build a flowinjection-analysis system in many biochemical research studies, but the viscous damping in liquids would cause not only large frequency shifts but also large losses in the quality factor $Q$ leading to instability and even cessation of oscillation. As a consequence, a QCM with dissipation (QCM-D) was developed for detection in liquid environments, ${ }^{(14-16)}$ but its complex operation process and high costs prevented it from being widely used. Therefore, in many research studies, QCMs were loaded with a droplet of liquid instead of being immersed in a sample liquid. ${ }^{(17-22)}$

The frequency shift caused by a localized or nonuniform mass attached to the QCM electrodes is given by ${ }^{(23)}$

*Corresponding author: e-mail: xianhehuang@uestc.edu.cn http://dx.doi.org/10.18494/SAM.2017.1434 


$$
\Delta f=\frac{1}{\pi r_{d}^{2}} \int_{0}^{2 \pi} \int_{0}^{r_{d}} \Delta m S_{f}(r, \theta) r d r d \theta
$$

where $S_{f}(r, \theta)$ is the mass sensitivity function, in hertz per kilogram, $\Delta m$ is the effective added mass, $r_{d}$ is the radius for the mass deposit on the electrode, and $r$ and $\theta$ are the polar coordinates of the point at which the mass is added.

QCMs are sensitive to the viscosity and density of the contacting liquid. In liquid, the effective mass $\Delta m$ is related to the liquid decay length $\delta$ when the QCM is covered by a viscoelastic film. ${ }^{(13,23,24)}$ The liquid decay length $\delta=\sqrt{\eta / \pi \rho f_{0}}$, where $f_{0}$ is the operating frequency of the QCM, and $\rho$ and $\eta$ are the density and viscosity of the liquid, respectively.

As shown in Fig. 1, when a small single drop of liquid (in microliter level) is loaded on the center of the electrode, the frequency shift of the QCM is caused by only the effective mass layer instead of the total mass of the liquid droplet. In general, the decay length of a liquid is very small (in micrometer level, far less than that shown in Fig. 1), so $\left(r_{d}-r_{a}\right)$ is also in micrometer level, that is, the radius of the effective mass layer can be considered as approximately equal to the droplet radius.

When a small single drop of liquid (in microliter level) is loaded on the center of the electrode, as shown in Fig. 1, the effective mass that causes the frequency shift of the QCM could be obtained as

$$
\Delta m=\frac{1}{2} r_{d}^{2} \sqrt{\pi \rho \eta / f_{0}}
$$

Josse et al. showed that the mass sensitivity function $S_{f}(r, \theta)$ of a QCM can be represented as ${ }^{(25)}$

$$
S_{f}(r, \theta)=\frac{|A(r)|^{2}}{2 \pi \int_{0}^{\infty} r|A(r)|^{2} d r} \times C_{f},
$$

where $A(r)$ is the particle displacement amplitude function, ${ }^{(25-27)} r$ is the distance from the center, and $C_{f}$ is Sauerbrey's sensitivity constant, with a value of $1.78 \times 10^{11} \mathrm{~Hz} \cdot \mathrm{cm}^{2} / \mathrm{kg}$.

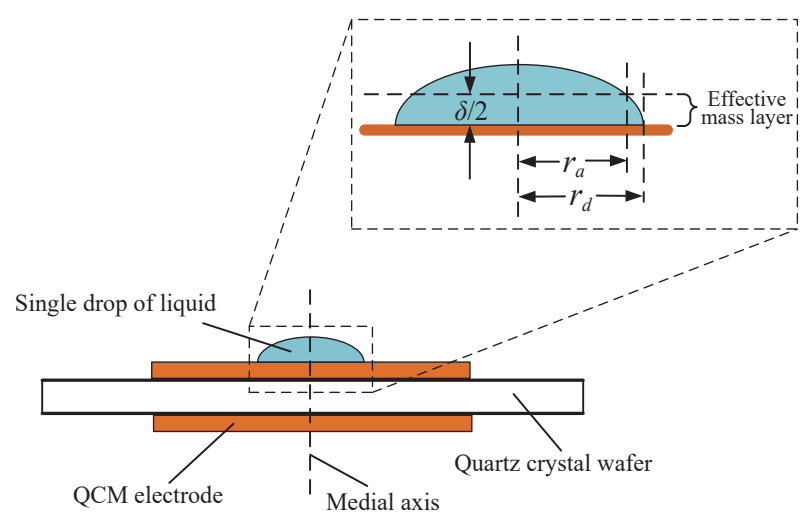

Fig. 1. (Color online) Schematic view of a single drop of liquid localized on the QCM electrode. 
The $S_{f}(r, \theta)$ of QCM could be calculated according to the above-mentioned analysis. Take the AT-cut $10 \mathrm{MHz}$ fundamental QCMs used in this study for example: the diameters of the crystal wafer and gold electrode are 8.7 and $5.1 \mathrm{~mm}$, and the thicknesses of the gold electrodes and quartz crystal wafer are about $1000 \AA$ and $0.167 \mathrm{~mm}$, respectively. The profile of mass-sensitivity distribution can be obtained as shown in Fig. 2.

Thus, the frequency shift of a QCM could be calculated as

$$
\Delta f=-\frac{1}{2} \sqrt{\rho \eta / \pi f_{0}} \int_{0}^{2 \pi} \int_{0}^{r_{d}} S_{f}(r, \theta) r d r d \theta .
$$

In summary, the frequency response of a QCM loaded with a droplet depends only on the radius of the liquid droplet, the operating frequency of the QCM, and the density and viscosity of the liquid.

\section{Methods}

An experiment was designed to verify this conclusion. At room temperature (about $25{ }^{\circ} \mathrm{C}$ ), a single drop of an organic reagent was loaded onto the center of the QCM electrode using a microinjector: the radius of the droplet increases with time, as shown in Fig. 3. Then, the response of the QCM and the radius of the droplet were measured with a vector network analyzer (Agilent E5062A) and a tool microscope (TM-505) every 5 min until the radius stopped increasing.

Figure 4 shows a schematic of the overall experimental setup. The microinjector was purchased from Shanghai Gaoge Industrial and Trading Co., Ltd. (Shanghai, China). The tool microscope was purchased from Shanghai BM Optical Instruments and Manufacturing Co., Ltd. (Shanghai, China). The QCM was purchased from Tongfang Guoxin Electronics Co., Ltd. (Hebei, China). The organic reagent (lubricating oil OW-40) was purchased from BP Corporate Introduction, and its density and viscosity are $\rho=0.842 \times 10^{3} \mathrm{~kg} / \mathrm{m}^{3}$ and $\eta=110 \mathrm{mPa} \cdot \mathrm{s}$ at room temperature $\left(25^{\circ} \mathrm{C}\right)$, respectively. Thus, its liquid decay length could be calculated at $4.16 \mu \mathrm{m}$.

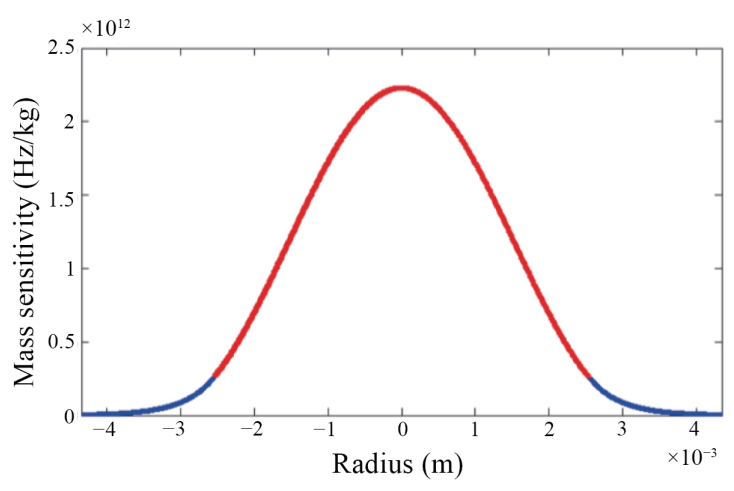

Fig. 2. (Color online) Mass-sensitivity distribution for AT-cut $10 \mathrm{MHz}$ fundamental QCM. The red line represents the electrode region $(|r|<2.55 \mathrm{~mm})$.

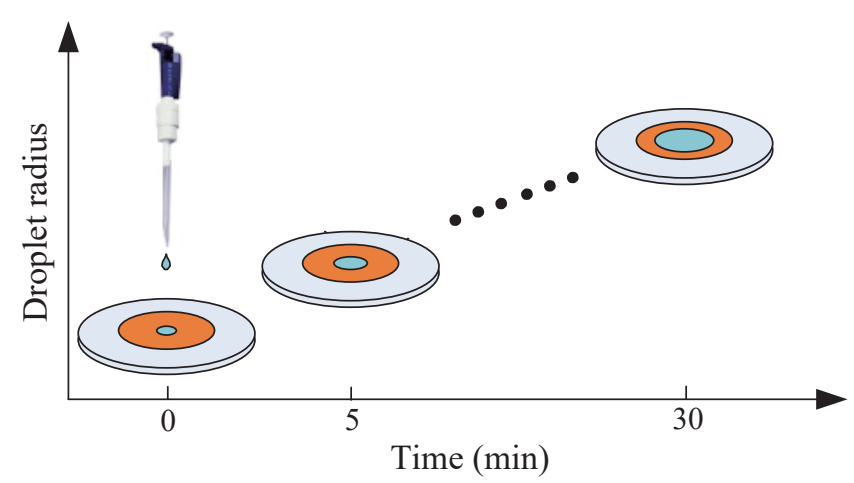

Fig. 3. (Color online) The radius of the droplet on the electrode increases with time. 


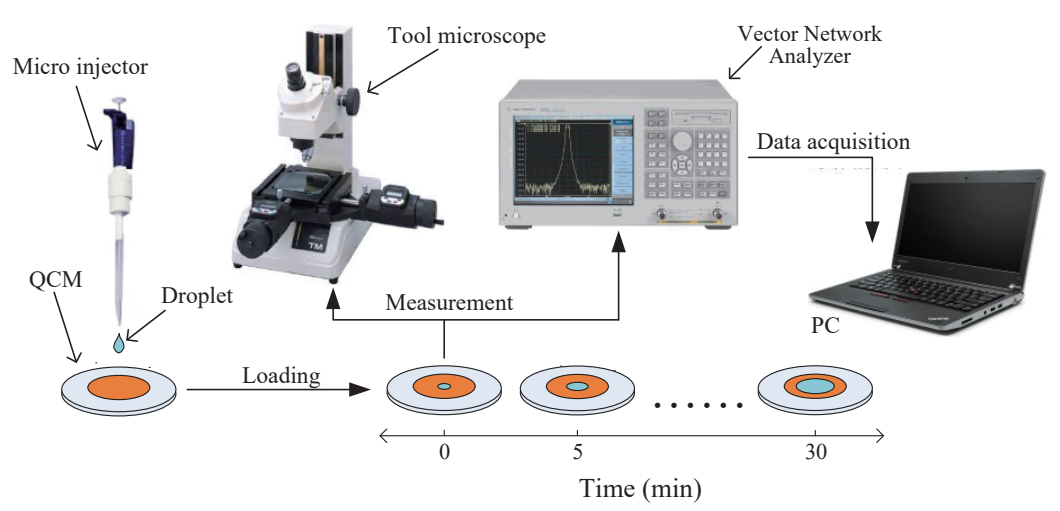

Fig. 4. (Color online) A schematic of the overall experimental setup.

\section{Results and Discussion}

The response of the QCM without loading is shown in Fig. 5. The response of the QCM with loading (as shown in Fig. 6) and the radius of the droplet (as shown in Fig. 7) were measured every $5 \mathrm{~min}$. The minimum reflection frequency (the frequency corresponding to the minimum S11 parameter) of the QCM without loading is $9989225 \mathrm{~Hz}$. Then a drop of sample liquid (in microliter level) was loaded on the center of the QCM electrode. The initial frequency shift caused by the droplet and the initial radius of the droplet were measured at $2475 \mathrm{~Hz}$ and $0.68 \mathrm{~mm}$, respectively.

As expected, the droplet radius increased over time, and the growth trend gradually slowed down. In the meantime, a similar increase in frequency shift was found. The droplet radius and frequency shift almost remained constant until 30 min later.

As can be seen in Figs. 6 and 7, there was no change in the total mass of the droplet, but the experimental frequency shift increases with the increase in radius over time. Why does this happen? Although the total mass of the droplet did not change, the effective mass increases quadratically as the radius increases. Consequently, the frequency shift of the QCM showed a significantly quadratic increase.

Eight sets of data were measured: the corresponding theoretical frequency shift was also calculated according to Eq. (4) and then compared with the experimental frequency shift shown in Fig. 7. The absolute values of errors between the theoretical and experimental frequency shifts are small, so the experimental data can be considered in accordance with the theoretical inferences.

In this study, the liquid decay length is very small $(4.16 \mu \mathrm{m})$ so the errors caused by the contact angle problem could be ignored. However, there is a phenomenon worthy of attention: the errors between the experimental and theoretical frequency shift were increased while the droplet radius increased over time. Thus, we assume that the contact angle will decrease over time and cause larger errors. Therefore, further study of contact angle is significant for the further understanding of the effective mass layer of a single drop of liquid located on a QCM, and a related study is already being planned. 


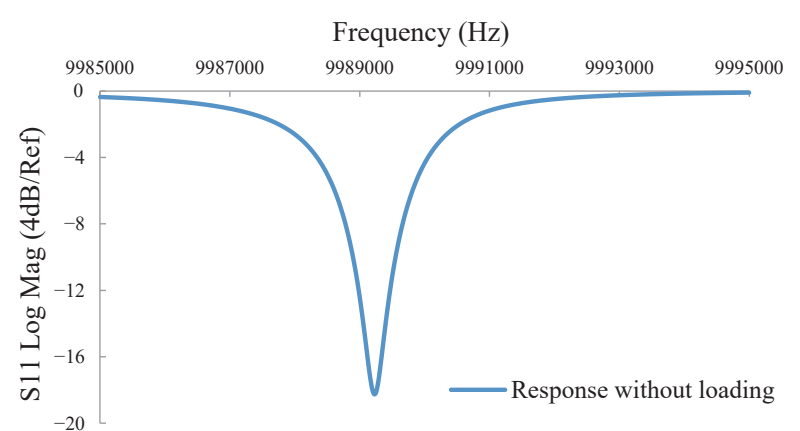

Fig. 5. (Color online) Response of QCM without loading.

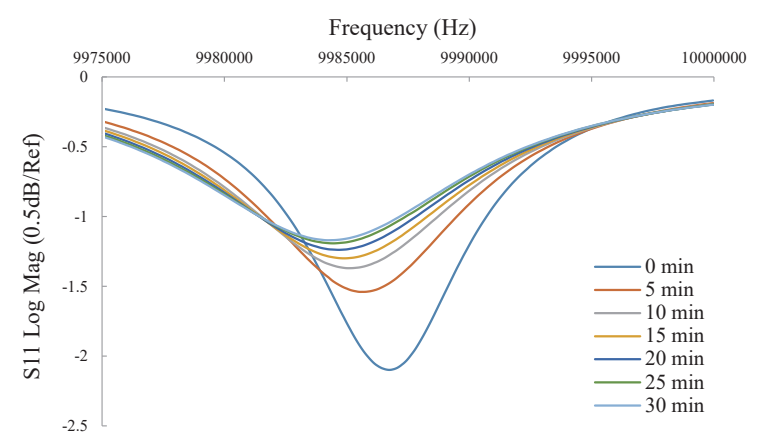

Fig. 6. (Color online) Response of QCM with loading changed over time (measured every $5 \mathrm{~min}$ ).

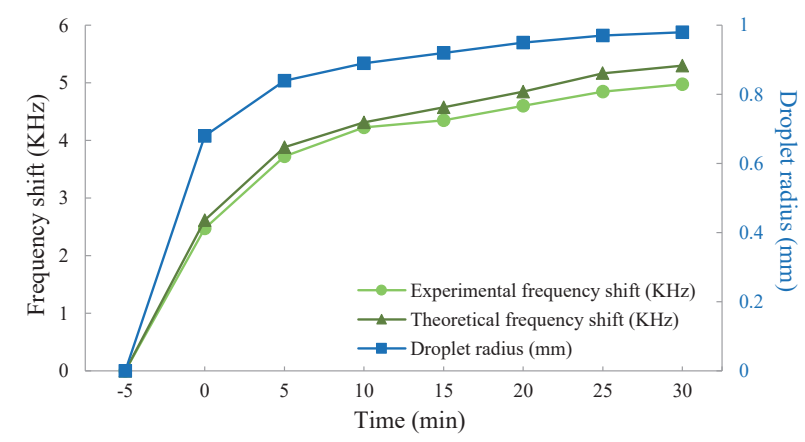

Fig. 7. (Color online) Droplet radius and experimental/theoretical frequency shift of the QCM.

\section{Conclusions}

By theoretical and experimental analyses, we found that the frequency response of a quartz crystal microbalance loaded with a single drop of liquid depends on only the effective mass layer, which is relevant to the liquid decay length and the radius of the droplet, and the liquid decay length is determined using the density and viscosity of the liquid and the operating frequency of the QCM. A model that describes the frequency response of the QCM was established, and a novel verification experiment was designed and performed, which shows that this model is valid.

\section{References}

1 J. X. Liang, J. Huang, S. Ding, and T. Ueda: Sens. Mater. 25 (2013) 519.

2 Z. Parlak, C. Biet, and S. Zauscher: Meas. Sci. Technol. 24 (2013) 085301.

3 X. H. Wang, Y. L. Yao, J. Zhang, Z. Q. Zhu, and J. Z. Zhu: Sens. Mater. 20 (2008) 111.

4 Y. Dehari, Y. Takei, S. Koyama, H. Nanto, and S. Seki: Sens. Mater. 22 (2010) 175.

5 D. Lee and T. H. Cui: IEEE Sensors J. 9 (2009) 449.

6 T. P. Huynh and W. Kutner: Biosens. Bioelectron. 74 (2015) 856.

7 A. Mirmohseni, R. Pourata, and M. Shojaei: IEEE Sens. J. 14 (2014) 2807. 
8 Z. P. Yang and C. J. Zhang: Sens. Actuators, B 142 (2009) 210.

9 H. F. El-Sharif, H. Aizawa, and S. M. Reddy: Sens. Actuators, B 206 (2015) 239.

10 H. Matsumoto, M. Matsuoka, S. Iwamori, and K. Noda: Sens. Mater. 22 (2010) 61.

11 E. Juzeliunas: Chemija 20 (2009) 218.

12 G. Sauerbrey: Z. Phys. 155 (1959) 206.

13 K. K. Kanazawa: Faraday Discuss. 107 (1997) 77.

14 N. J. Cho, C. W. Frank, B. Kasemo, and F. Hook: Nat. Protoc. 5 (2010) 1096.

15 X. Turon, O. J. Rojas, and R. S. Deinhammer: Langmuir 24 (2008) 3880.

16 M. Hussain, H. Northoff, and F. K.Gehring: Biosens. Bioelectron. 66 (2015) 579.

17 Y. J. Chang and Y. J. Huang: Sens. Actuators, B 222 (2016) 682.

18 L. Yang, X. H. Huang, L. Sun, and L. Xu: Sens. Actuators, B 224 (2016) 863.

19 H. Zhuang, P. Lu, S. P. Lim, and H. P. Lee: Anal. Chem. 80 (2008) 7347.

20 J. Watanabe, H. Y. Shen, and M. Akashi: Acta Biomater. 4 (2008) 1255.

21 H. Zhuang, P. Lu, S. P. Lim, and H. P. Lee: Langmuir 24 (2008) 8373.

22 Q. S. Bai and X. H. Huang: J. Sens. (2016) Article ID: 7580483.

23 A. C. Hillier and M. D. Ward: Anal. Chem. 64 (1992) 2539.

24 S. J. Martin, V. E. Granstaff, and G. C. Frye: Anal. Chem. 63 (1991) 2272.

25 F. Josse, Y. Lee, S. J. Martin, and R. W. Cernosek: Anal. Chem. 70 (1998) 237.

26 J. Y. Gao, X. H. Huang, and Y. Wang: IEEE Trans. Ultrason. Ferroelectr. Freq. Control 60 (2010) 1883.

27 P. J. Cumpson and M. P. Seah: Meas. Sci. Technol. 1 (1990) 544. 